\title{
Deformation of Single Crystal Molybdenum at High Pressure
}

Brian P.Bonner, Chantel Aracne, Daniel L. Farber, Carl O. Boro, David Lassila

February 19, 2004 


\section{Disclaimer}

This document was prepared as an account of work sponsored by an agency of the United States Government. Neither the United States Government nor the University of California nor any of their employees, makes any warranty, express or implied, or assumes any legal liability or responsibility for the accuracy, completeness, or usefulness of any information, apparatus, product, or process disclosed, or represents that its use would not infringe privately owned rights. Reference herein to any specific commercial product, process, or service by trade name, trademark, manufacturer, or otherwise, does not necessarily constitute or imply its endorsement, recommendation, or favoring by the United States Government or the University of California. The views and opinions of authors expressed herein do not necessarily state or reflect those of the United States Government or the University of California, and shall not be used for advertising or product endorsement purposes.

\section{Auspices Statement}

This work was performed under the auspices of the U.S. Department of Energy by University of California, Lawrence Livermore National Laboratory under Contract W-7405-Eng-48. 
Deformation of Single Crystal Molybdenum at High Pressure

Brian P. Bonner ${ }^{1}$, Chantel Aracne ${ }^{1}$, Daniel L. Farber ${ }^{1}$, Carl O. Boro ${ }^{1}$, David Lassila ${ }^{2}$

Energy and Environment ${ }^{1}$ and Engineering ${ }^{2}$ Directorates, Lawrence Livermore National Laboratory, Livermore CA, 94550

Single crystal samples of micron dimensions oriented in the [001] direction were shortened 10 to $40 \%$ in uniaxial compression with superposed hydrostatic pressure to begin investigation of how the onset of yielding evolves with pressure. A testing machine based on opposed anvil geometry with precision pneumatic control of the applied force and capability to measure sub micron displacements was developed to produce shape changing deformation at pressure. The experiments extend observations of pressure dependent deformation to $\sim 5 \mathrm{Gpa}$ at shortening rates of $\sim 2 * 10^{-4}$. Samples have been recovered for post run characterization and analysis to determine if deformation mechanisms are altered by pressure.

Experiments under hydrostatic pressure provide insight into the nature of materials under extreme conditions, and also provide a means for altering deformation behavior in a controlled fashion. The approach has a long history ${ }^{1,2}$ demonstrating that pressure enhances ductility in general, and produces enhanced hardening relative to that expected from normal cold work in the BCC metals $\mathrm{Mo}$, Ta and $\mathrm{Nb}^{2}$. The pressure hardening is in excess of that predicted from the measured increase in shear modulus at pressure, and therefore is likely due to a dislocation mechanism, such as suppression of kink pair formation or the interaction of forest dislocation cores, and not from lattice resistance. The effect has not been observed in FCC metals, suggesting a fundamental difference between deformation mechanisms at pressure for the two classes.

The purpose of this letter is to investigate the origin of pressure hardening with new experiments that extend the pressure range beyond $3 \mathrm{GPa}$, the upper limit of conventional large sample $\left(1 \mathrm{~cm}^{3}\right)$ testing methods ${ }^{2}$. Most previous high pressure deformation studies have been on poly crystals, relying on model dependent analysis to infer the maximum deviatoric stress that a deformed sample can support ${ }^{3,4}$. In one experiment ${ }^{5}$, a single crystal of the silicate olivine was compressed at $16 \mathrm{GPa}$ with a sapphire uniaxial stress gage in the sample chamber. Splitting of the ruby fluorescence line increases with deviatoric stress, enabling direct measurement of the sample stress. Unfortunately, this method is not sensitive enough to determine first yielding of [001] single crystal Mo, and was not used in this study.

The method of this letter is fundamental. Data for the pressure dependence of single crystal elastic constants of molybdenum (for the same single crystals used in this study) recently determined by inelastic $\mathrm{x}$-ray scattering ${ }^{6}$ established a baseline for elastic deformation. The stress at first yield can then be calculated from a precise determination of the sample shortening as deformation proceeds under pressure. The sample itself 
provides the stress measurement. A new testing machine, based on the opposed anvil geometry (gem quality diamond anvils; 750 micron culets), was constructed for these experiments (Figure 1). The critical additions for controlled deformation are programmable pneumatic control of the applied force, and high resolution monitoring of the advance of the tungsten carbide backing plates that support the anvils to measure sample shortening. The actuator is controlled by an electronic valve/regulator that stabilizes pressure to $\sim 0.5 \mathrm{psi}\left(3.44 * 10^{-3} \mathrm{MPa}\right)$ with proper choice of the PID parameters. Displacements are measured by a commercially available noncontact fiber optic system with a factory calibrated sensitivity of 0.02 microns that was checked by comparison with a precision digital micrometer.

The experimental procedure is as follows. First, a BeCu gasket, initially 250 microns thick, is precompressed between the anvils while the force and indentation displacement are monitored until the desired depth is reached. Several candidate gasket materials were evaluated. $\mathrm{Be} \mathrm{Cu}$ was most suitable for measurements below $10 \mathrm{GPa}$. If the indentation behavior is anomalous, the gasket is discarded because successful execution depends on smooth, predicable gasket deformation. A pressure chamber is then formed in the gasket by electrical discharge machining (EDM). The micro sample (Figure 2a,b), prepared by EDM or femto second laser cutting to form the cylinder, and abrasive and electro polishing to thin to final height, is then loaded into the pressure chamber (Figure 2c) together with a methanol-ethanol-water (16:3:1) pressure transmitting fluid. This fluid remains liquid to approximately $10 \mathrm{GPa}$. The difference in height between the preindented gasket and sample controls the pressure at contact, and thus the applied pressure during deformation. Pressure increases somewhat during the experiment as the anvils advance and the sample shortens, and the increase is determined independently by ruby fluorescence measurements.

A control experiment was performed at ambient pressure on a sample 504 microns in diameter and 194 microns high, in a 661 micron pressure chamber. The complete load displacement curve is plotted in Figure 3a. At first, the reloaded $\mathrm{BeCu}$ gasket responds elastically and then yields. Approximately 25 microns after the beginning of reloading, the anvil contacts the Mo sample and the load curve dramatically stiffens as the high modulus Mo sample is stressed. The ratio of areas of sample to gasket supporting the anvil is approximately $2 / 1$. An expanded view of initial sample loading is given as Figure 3b. Taking the linear incompressibility (modulus) in the [001] direction to be $400 \mathrm{GPa}$, and a measured compressive strain on the sample of $2.0 * 10^{-3}$, the upper limit on stress the sample supports is $0.82 \mathrm{GPa}$. Since first yield (generation of Frank Read dislocation sources) occurs for this orientation near strains of $3.0^{*} 10^{-4}$, the strain resolution of this data set is insufficient to improve the estimate of first yield.

Experimental results at $\sim 4.5 \mathrm{GPa}$ are presented in Figure 4a,b. The initial sample dimensions were 300 by 195 microns. The pressure chamber was initially 525 microns in diameter and grew to 566 microns after the experiment. The loading history is more complicated than in the first experiment. It is likely that sample contact did not occur until approximately 50 microns into reloading when the load displacement curve stiffens. Immediately after the load displacement curve turned upward, time dependent flow 
began, suggesting that the gasket and sample had yielded. Slight stiffening of the loading curve after 10 to 15 microns of indentation may indicate earlier contact, but this is unlikely. The small sample/gasket area ratio for a 300 micron sample made detection of contact problematic with the displacement sensitivity used in this experiment. Shape changing deformation to a longitudinal strain of 0.38 did occur, as is clearly seen in pre and post experiment photomicrographs (Figure $5 \mathrm{a}, \mathrm{b}$ ). Deformation produces the characteristic shape expected for slip on [111] planes for cubic symmetry.

In summary, shape changing deformation has been achieved on samples of Mo oriented for compression in the [001] direction with simultaneous superposed hydrostatic pressure of $\sim 4.5 \mathrm{GPa}$. This pressure is $150 \%$ higher than any reported in the literature for deformation of single crystal metals. Recovered samples are of sufficient quality for post run characterization and studies of deformation mechanism. The testing machine constructed for these measurements is capable of pressures in the 10-30 GPa range and minor improvements in technique will make future measurements more quantitative.

Acknowledgements. The authors thank F. Occelli, M. LeBlanc, D. Ruddle and B. Olsen for useful discussions and assistance. The work was funded by the LLNL LDRD program and was conducted under the auspices of the U.S. Department of Energy by University of California, Lawrence Livermore National Laboratory under Contract W-7405-Eng-48.

${ }^{1}$ T. Von Karman, Forschungheft, 118 and Z. Ver.Deut.Ing (1911).

${ }^{2}$ P.W. Bridgman, J. Appl. Phys. 24, 560 (1953).

${ }^{3}$ C. Meade and R. Jeanloz, J. Geophys. Res. 93, 3261 (1988).

${ }^{4}$ T.Duffy, G. Shen, H.K. Mao,R. Hemley, and A. Singh, J. Appl. Phys. 86 12, 6729 (1999).

${ }^{5}$ M. Chai, J.M.Brown, and Y. Wang, in Properties of Earth and Planetary Materials at High Pressure and Temperature, eds., M.H. Manghnani and T. Yagi, American Geophysical Union 483 (1998).

${ }^{6}$ D. L. Farber, F. Occelli, J. Badro and M. Kriesch, unpublished manuscript, (2004). 


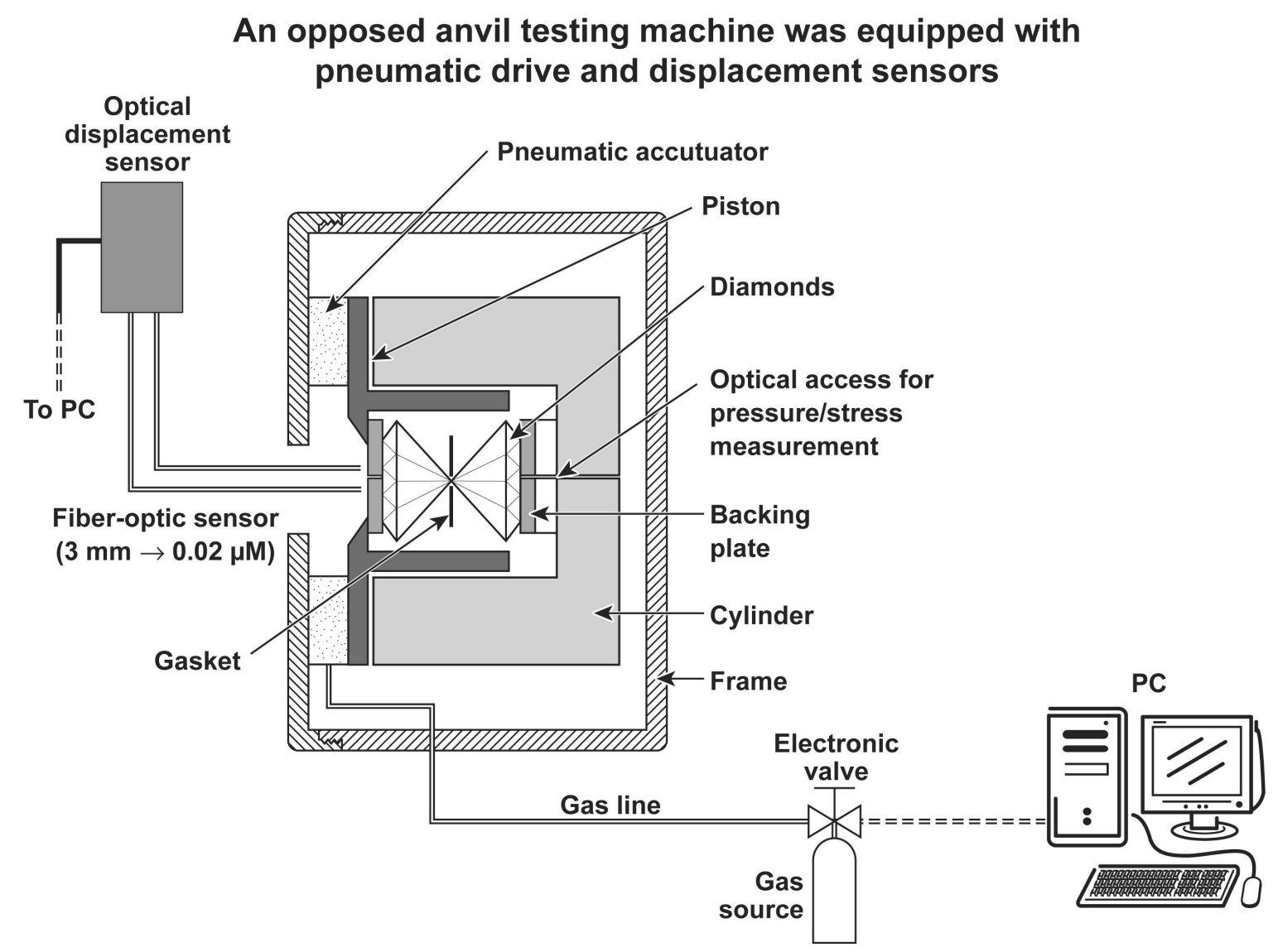

Figure 1. Schematic of the micro testing machine. 
Figure 2a. 300, 400 and 500 micron Mo [001] single crystals prepared for testing.

Figure 2b. Side view of the 195 by 300 micron sample.

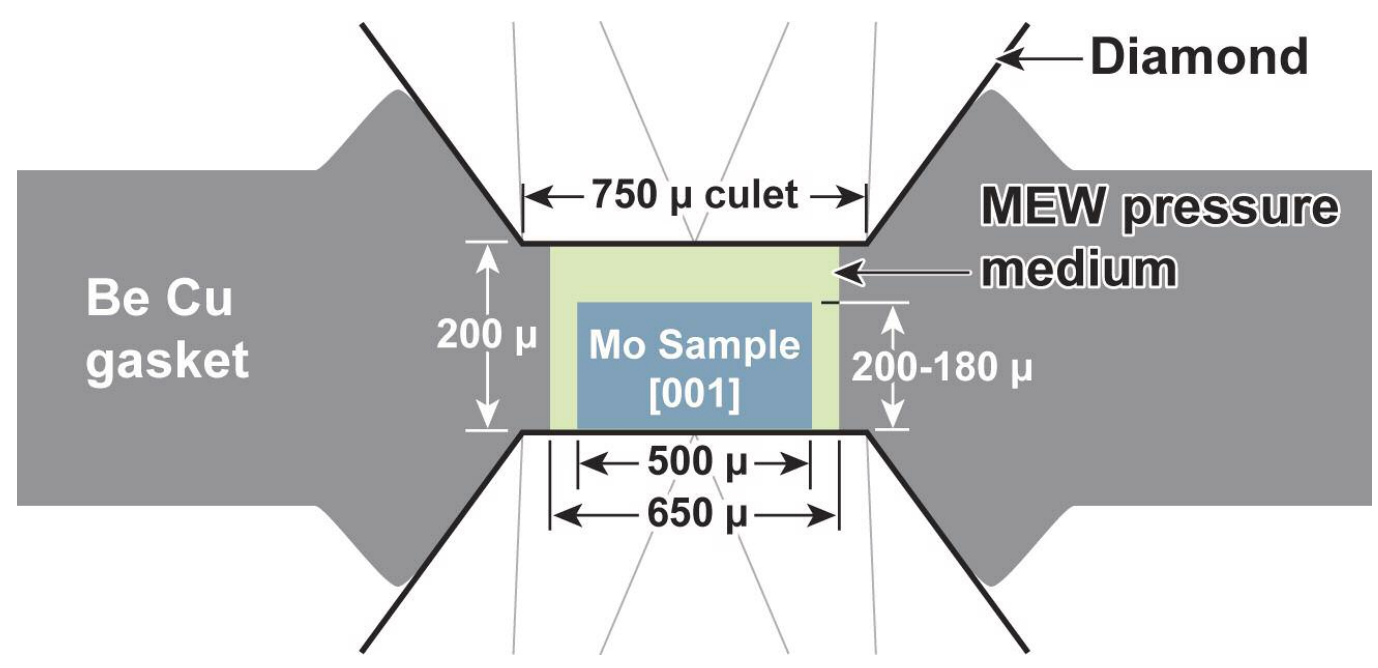

Figure 2c. Side view through the pressure chamber. 

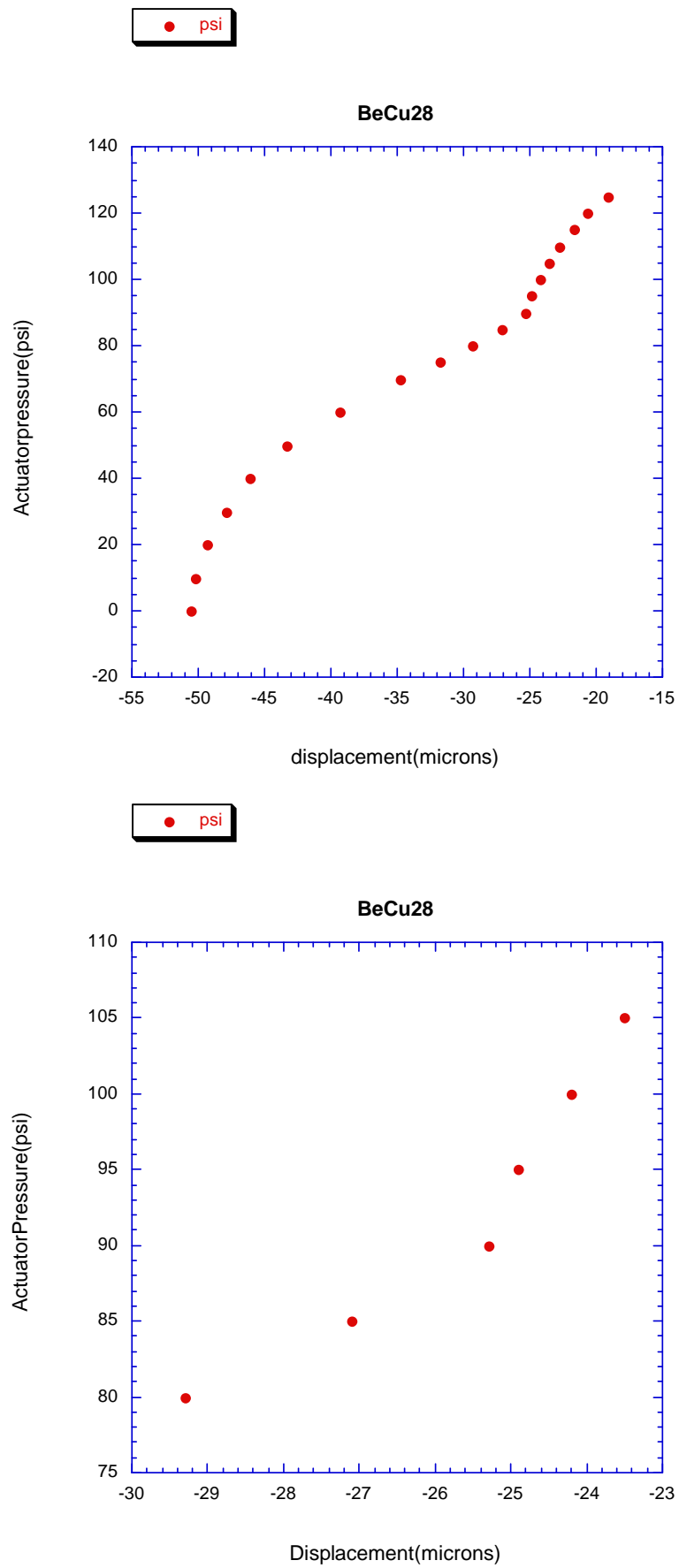

Figure 3 a,b. Load displacement history for a 500 micron Mo crystal deformed at ambient pressure. 

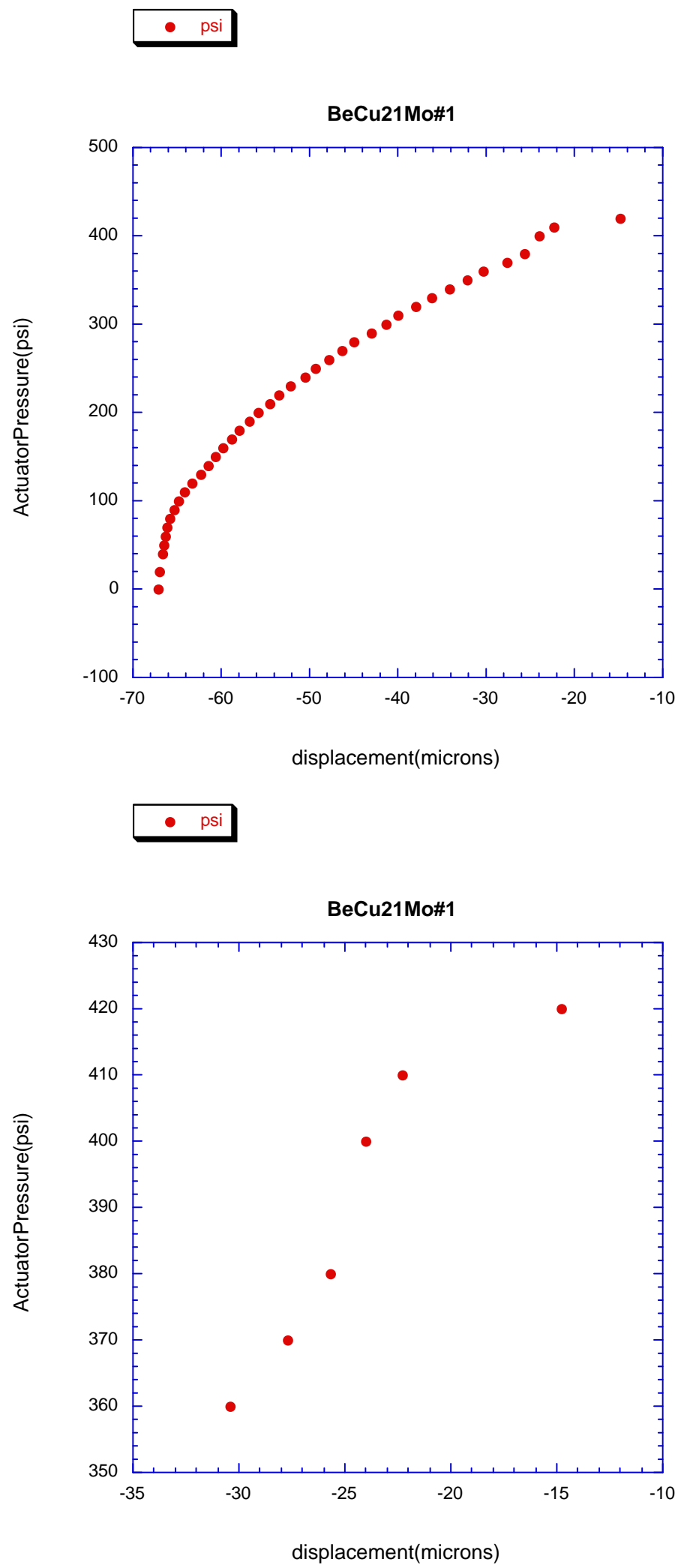
Figure 4a,b. Loading displacement history for a 300 micron Mo sample deformed at $~ 4.5$ GPa. 
Figure 5a,b. Before and after micrographs of Mo [001] 300 x 195 microns deformed at $\sim 4.5 \mathrm{GPa}$. Post deformation dimensions are $244 \times 263$ along the diagonals on the polished surface and 121 microns thick.

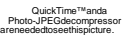


